Gut, 1986, 27, S1, 14-17

\title{
Indications for nutritional support
}

\author{
Y A CARPENTIER \\ Clinical Nutrition Unit, Hôpital Saint Pierre, Free University of Brussels, Belgium
}

SUMMARY In this review different types of patients who usually require nutritional support are considered and recommendations made as to when nutritional support should be started, how long it should be continued, and which substrates should be preferentially used by which route.

The resolution of many technical problems and the development of new products have considerably improved the safety and the efficacy of both enteral and parenteral nutrition. Despite our rapidly expanding knowledge, however, the indications for nutritional support have not been clearly defined. This is largely due to the relative paucity of clinical data available from well designed controlled studies which make it difficult to determine the possible benefits of nutritional support in many situations.

\section{Patients requiring nutritional support}

A high proportion of patients present, at the time of admission to hospital, with a severely impaired nutritional state that is unlikely to improve spontaneously in the near future. Others present at admission with a normal or slightly impaired nutritional state, which deteriorates during hospital stay as a consequence of inadequate nutritional intake, or medical or surgical treatment, or a combination of all three. Patients in need of nutritional support usually fall into four categories.

\section{PATIENTS WITH SEVERELY IMPAIRED \\ GASTROINTESTINAL FUNCTION}

This may occur as a consequence of massive small bowel resection, high output fistulae of the small intestine, subacute intestinal obstruction, prolonged ileus, severe malabsorption, widespread small bowel Crohn's disease, and complicated and prolonged episodes of acute pancreatitis.

2 PATIENTS WITH INADEQUATE FOOD INTAKE In one group of patients oral intake is insufficient to keep pace with the increased energy expenditure arising as a consequence of extended burns, major trauma, severe and prolonged sepsis, and severe and extensive conditions of inflammatory bowel disease.

Correspondence to: Professor Y A Carpentier. Clinical Nutrition Unit. Hôpital Universitaire Saint Pierre, Rue Haute 322, $10(0)$ Bruxelles. Belgium.
In other patients normal energy requirements cannot be met because of persisting nausea or vomiting (due to the presence of a tumour or central nervous system lesions, or as a side effect of drug treatment), central nervous system lesions (stroke, coma, neurosurgery), or psychological factors (severe anorexia nervosa, depression). In these patients it is clearly important to monitor the extent of difference between intake and output so that by giving nutritional support early a rapid deterioration in nutritional state can be prevented.

\section{PATIENTS UNDERGOING MAJOR SURGERY}

A close association between the preoperative nutritional state and the outcome of surgical patients has been clearly established. More recently prognostic indices based on measurements of nutritional state have been developed. ${ }^{12}$ Although these indices have proved successful in predicting operative risks, it has recently been shown that similar results can be obtained using the simple determination of muscular function. ${ }^{34}$ Preoperative nutritional support has been shown to lower operative risks in patients presenting with a poor prognostic index on admission. ${ }^{5}$ Similarly, Muller $e t$ al $l^{6}$ purported to show that preoperative nutritional support in patients undergoing surgery for upper gastrointestinal tract cancer reduced postoperative morbidity and mortality. Interpretation of the results of the above study was impeded, however, by the fact that both well nourished and nutritionally depleted patients were included; the different operative procedures undertaken make it difficult to define clearly, on the basis of published results, the types of patients who would really benefit from preoperative support. Moreover, there are as yet no published data that provide guide lines as to how long preoperative nutritional support should be given.

Another problem that has arisen is the difficulty that occurs in making an accurate assessment of nutritional state after major injury. Anthropometric 
measurements do not take into account the relative increase in body water and therefore often underestimate nutritional depletion. Plasma protein concentrations are affected by increased pool sizes and are modified by inflammation. ${ }^{7}$ Unfortunately, techniques for precisely measuring body composition are available only in research units and are therefore not applicable to routine clinical practice. Therefore, in the present state of the art the decision to initiate preoperative nutritional support should be taken on an individual basis.

4 PATIENTS WITH CANCER

Only patients with potentially treatable disease should be considered. The interference of the treatment (surgery, radiation, chemotherapy) with food intake and energy balance should be taken into account. No clear indication that nutritional support prolongs survival has been shown, and the possibility that nutritional support may improve the general condition, and therefore the quality of life, has not yet been analysed.

\section{Aims of nutritional support}

Once a patient has been considered to be a suitable candidate, it is necessary to define the aims of nutritional support on an individual basis. In nonhypermetabolic patients with gastrointestinal failure nutritional support can be started on a temporary or permanent basis and may represent a partial or complete substitute for oral feeding.

The aim of nutritional support in depleted patients should be to restore normal body composition. Often this cannot be reached within a short period of time, and, therefore, priorities in the areas to be replenished should be defined. In hypermetabolic patients attempts to compensate for increased losses that are too rapid will result in excessive intake, which in turn is likely to lead to severe and undesirable side effects. It is important to appreciate that during the period of changed metabolic response to substrate intake the primary aim of nutritional support should be to minimise nutrient losses.

\section{When should nutritional support be started?}

As there seems to be a clear association between the degree of weight loss and outcome ${ }^{\gamma}$ it becomes important to consider the influence of various factors on weight loss as well as the actual composition of the weight loss. In non-traumatised subjects submitted to complete starvation initial weight loss is pronounced but stabilises after a few days; by that time the contribution of protein and fat to lost weight is also constant (about $12.5 \%$ protein and $25 \%$ fat). Fig. 1 shows that even ingestion of small amounts of food can very efficiently limit the rate and extent of weight loss. ${ }^{1011}$

Hypermetabolic, injured, or septic patients undergoing complete starvation lose about twice as much weight as normal subjects. Administration of food equivalent to $40-60 \%$ of estimated requirements can reduce weight loss by about half. Recently Kinney reported data on the composition of lost weight in totally starved and partially fed hypermetabolic patients ${ }^{11}$ and compared them with previous data on normal subjects published by others. ${ }^{10} \mathrm{He}$ pointed out that the proportion of protein in lost weight was comparable in these different conditions and that food intake influenced fat losses to a much greater extent than protein losses (Fig. 2).

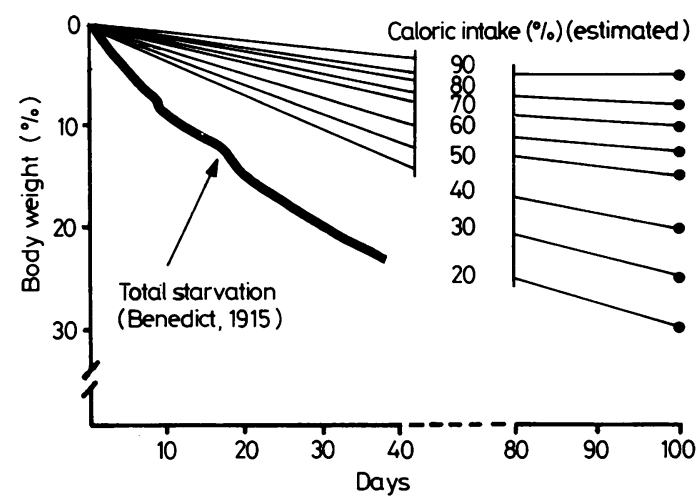

Fig. 1 Weight loss in normal subjects undergoing either total starvation $(n=9)$ or partial calorie intake $(n=10) .{ }^{1 l}$

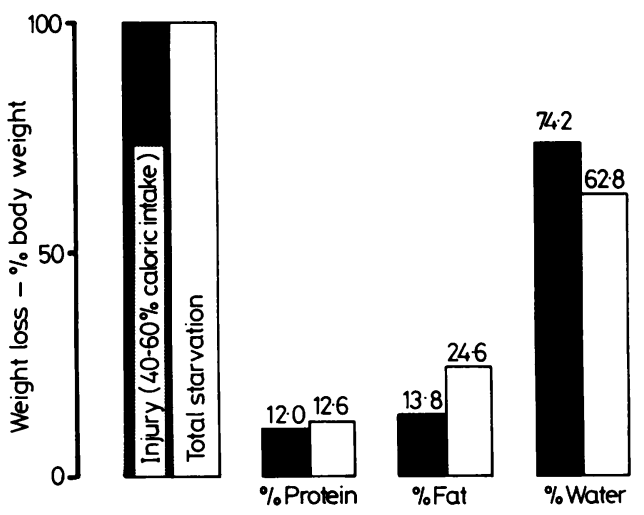

Fig. 2 Tissue composition of weight loss in normal subjects submitted to total starvation and severely injured patients receiving partial calorie intake. " 
As a consequence of these results, I strongly recommend that nutritional support is not unduly delayed, particularly when it is clear from experience that nutritional support will be required at some stage. This is particularly relevant in patients with a high energy expenditure, or previous nutritional depletion, or both, even if only a fraction of the estimated requirements can be safely provided (that is, during the early postoperative phase).

\section{How long should nutritional support be continued?}

In non-surgical patients nutritional support has to be continued until the patient is able to compensate for his or her expenditure-or to replete his or her losses-by normal oral feeding. This should be assessed by precise dietetic measurements. As mentioned above, in patients receiving preoperative nutrition there is no available variable to indicate when surgical risks have been greatly reduced. The prognostic indices, mainly based on slow turnover proteins, albumin, and transferrin are not improved by limited periods of nutritional support. Therefore, although they can help predict surgical risks on admission, they cannot indicate when preoperative nutrition has reached its supposed goal.

Five days of preoperative nutritional support seem to be enough to improve greatly, and even normalise, the amount of fast turnover proteins such as thyroxin binding prealbumin and retinol binding protein. ${ }^{7}$ Impaired lymphocyte and polymorphonuclear leucocyte function, however, resulting from malnutrition, require at least two weeks of nutritional treatment to be restored. ${ }^{12}$ The association between the normalisation of these various variables and the postoperative outcome has still to be determined.

\section{Optimal substrate intake and route of administration}

Accurate measurements of energy expenditure by indirect calorimetry have shown that in most clinical conditions daily requirements rarely exceeded 2000-2500 kcal. These values are far lower than those reported even a decade ago. Except for extensive burn injuries, multiple fractures, and some acute septic episodes, energy expenditure is usually more influenced by the physical state, age, and sex of a patient than by the disease ${ }^{1314}$ and, therefore, can be grossly estimated using equations developed by Harris and Benedict ${ }^{15}$ or Consolazio and Johnson. ${ }^{16}$ It should also be remembered that depleted patients have a low energy expenditure and a reduced metabolic response to injury or sepsis. ${ }^{17}$

Intravenous administration of high calorie regimens to hypermetabolic patients can induce severe side effects, especially when glucose is the major or virtually unique calorie source (Table 1 ). ${ }^{18}$ The total calorie intake should therefore be limited during the hypermetabolic or "flow" phase. Plasma glucose and lipid (triglyceride, phospholipid, and cholesterol) concentrations should be monitored before and during parenteral nutrition, and the amount of infused fat and carbohydrate based on the individual tolerance to each substrate. Indeed, too fast or excessive administration of fat emulsion can also be associated with complications (Table 2). Although objective data resulting from well controlled studies are scarce, it seems reasonable to follow similar guidelines when enteral nutrition is administered to hypermetabolic subjects.

Complications are less likely to occur when nutritional support is given to untraumatised patients; great caution, however, is needed in refeeding severely depleted patients, and no advantage can be expected from excessive intake.

Parenteral and enteral nutrition are not competitive but complementary procedures. Enteral nutrition is associated with a lower cost and a reduced incidence of metabolic and septic complications. This route of administration is preferable whenever gastrointestinal function is sufficient. It is often

Table 1 Side effects of high glucose intake in hypermetabolic patients

Insulin resistance (high release of counterregulatory hormones) Limited inhibition of hepatic glucose production

High plasma glucose concentration and urinary glucose excretion Stimulation of glucagon and catecholamine release

Limited conversion to fat but glycogen deposition in muscle and liver

Increased oxygen consumption, carbon dioxide production, and minute ventilation

Limited inhibition of fat mobilisation and oxidation

Decreased plasma inorganic phosphate concentration

Table 2 Side effects resulting from exogenous fat infusions

In vitro agglutination of exogenous particles in the presence of $\mathrm{C}$ reactive protein rich and albumin poor plasma (relevance for in vivo use?)

Intracellular deposition of electron dense particles

Changed functions of polymorphonuclear leucocytes, monocytes, and macrophages

Increase in triglyceride, phospholipid, and free cholesterol plasma concentrations during rapid infusion

Modifications of the lipoprotein pattern and possible formation of abnormal particles

Interference with essential fatty acid and prostaglandin metabolism 
forgotten that there is an enormous reserve absorptive capacity of the small intestine so that enteral nutrition can often be successfully started, even when only a limited portion of the intestinal tract is functional.

The use of parenteral nutrition should be restricted to those patients whose digestive tract cannot or should not be used, or when it is impossible to meet the nutritional requirements by using the enteral route alone. In such cases parenteral nutrition must be combined and intake via the parenteral route must be adjusted in such a way that an optimal intake is provided by a combination of the two techniques.

These general guidelines should be considered with flexibility. For each individual case the final decision concerning the amount of nutritional support to be provided by each route has to take into consideration factors such as the degree of deterioration of the nutritional state, the functional absorptive capacities of the digestive tract, the effect of the primary underlying disease and of its treatment on energy expenditure and food intake, as well as the experience and facilities of the local unit.

\section{References}

1 Buzby GP, Mullen JL, Matthews DC, Hobbs CL, Rosato EF. Prognostic nutritional index in gastrointestinal surgery. Am J Surg 1980; 139: 160-7.

2 Simms JM, Smith JAR, Woods HF. A modified prognostic index based upon nutritional measurements. Clin Nutr 1982; 1: 71-9.

3 Klidjian AM, Foster KJ, Kammerling RM, Cooper AC, Karran SJ. Anthropometric and dynamometry indices and the relationship of post operative complications. $\mathrm{Br}$ Med J 1980; 281: 899-901.

4 Griffith CDM, Clark RG. A comparison of the "Sheffield" pronostic index with forearm muscle dynamometry in patients from Sheffield undergoing major abdominal and urological surgery. Clin Nutr 1984; 3: 147-52.

5 Mullen JL, Buzby GP, Matthews DC, Smale BF, Rosato EF. Reduction of operative morbidity and mortality by combined preoperative and postoperative nutritional support. Ann Surg 1980; 192: 6(14-13.

6 Müller JM, Brenner U, Dienst C, Pilchmaier $H$. Preoperative parenteral feeding in patients with gastrointestinal carcinoma. Lancet 1982; i: 68-71.

7 Carpentier YA, Bruyns J, Barthel J. Plasma proteins in nutritional assessment. Proc Nutr Soc 1982; 41: 405-17.

8 Studley HO. Percentage of weight loss: a basic indicator of surgical risk in patients with chronic peptic ulcer. JAMA 1936; 106: 458-60.

9 Benedict FG. A study of prolonged fasting. Washington: Carnegie Institution of Washington, 1915: 416. (No 203.)

10 Keys A, Brozek J, Henschel A, Mickelsen O, Taylor HL. The biology of human starvation. Vol 1. Minneapolis: University of Minnesota Press, 1950: 714-46.

11 Kinney JM. The tissue composition of surgical weight loss. In: Johnston IDA, ed. Advances in parenteral nutrition, Lancaster: MTP Press, 1978: 511-20.

12 Druart ML, Carpentier YA, Duchateau J. Effects of surgery and nutritional support on some lymphocyte and PMN leucocyte functions in man. Clin Nutr (In press.)

13 Wilmore DW. The metabolic management of the critically ill. New York: Plenum Medical Book Company, 1977: 31-7.

14 Kinney JM, Lister J, Moore FD. Relationship of energy expenditure to total exchangeable potassium. Ann NY Acad Sci 1963; 110: 711-20.

15 Harris JA, Benedict FG. Biometric studies of basal metabolism in man. Washington: Carnegie Institution of Washington, 1919. (No 279.)

16 Consolazio CF, Hohnson HL. Measurement of energy cost in human. Fed Proc 1971; 30: 1444-50.

17 Bastow D, Rawlings F, Allison SP. Benefits of supplementary tube feeding after fractured neck of femur: randomized control trial. $\mathrm{Br}$ Med J 1983; 287: 1589-92.

18 Robin AP, Carpentier YA, Askanazi J, Nordenström J, Kinney JM. Metabolic consequences of hypertonic glucose infusion: a review. Acta Chir Belg 1981; 2-3: 133-40. 